\title{
Simulation of Recycling Cable in Underwater Towed System
}

\author{
Chuanlong Liu \\ College of Marine \\ Northwestern Polytechnical \\ University \\ Xi'an, China, (86)15909291754 \\ liu2011100322@163.com
}

\author{
Yuwen Zhang \\ College of Marine \\ Northwestern Polytechnical \\ University \\ Xi’an,China, (86)13609110453
}

\author{
Xulong Yuan \\ College of Marine \\ Northwestern Polytechnical \\ University \\ Xi'an,China,(86)18691859360 \\ yuanxulong@nwpu.edu.cn
}

\begin{abstract}
A three-dimensions dynamic model of a typical underwater towed system which consists of the navigation body, the cable and the drag body is studied. In the model, the governing equations of cable system are established based on the multibody dynamics method. The cable is split up into a series of ball-and-socket rigid rods, which may have different physical properties. The results in the paper indicate the movement and ballistic characteristics of underwater towed system during the recycling which can be useful to engineering staff.
\end{abstract}

Keywords-undewater towed system; mathematical modeling; ballistic; coupled movement

\section{INTRODUCTION}

Underwater toewed system are fundamental tools for many marine applications which include naval defense, seabed mapping and ocean environmental measurements. In general, the underwater towed system consists of the navigation body, the cable and the drag body. In the process of design and application of underwater towed system, it is important to predict the performance of towed system. As for the underwater towed system, the key is the cable-modeling. The six-degrees-of-freedom equations of motion for the cable are a couple of highly nonlinear equations, therefore the cable is split up into a series of isometric ball-and-socket rigid rods. Along with the hydrodynamic model of the navigation body and the drag body and the connection between the three, it comes to a closed dynamic equations.

\section{DYNAMIC MODEL OF UNDERWATER TOWED SYSTEM}

\section{A. Coordinate system and posture angle}

Fig. 1 is the schematic of underwater towed system. In order to simplify the problem, the paper don't consider the reverse movement of the cable.

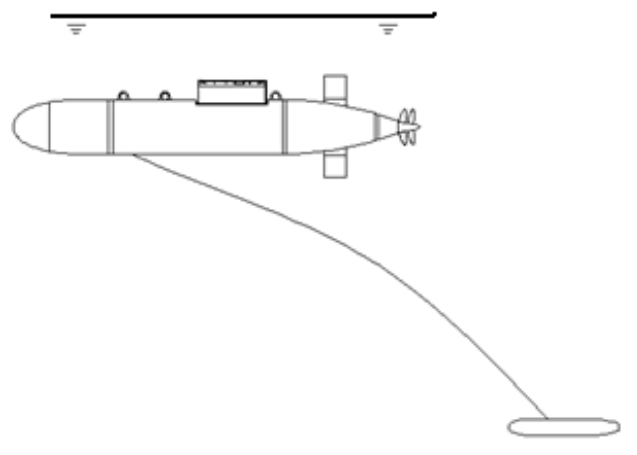

Figure 1. Underwater towed system

The model is defined in three coordinate systems: the inertial coordinate system $o_{0} x_{0} y_{0} z_{0}$, the body coordinate system $O x y z$ and the speed coordinate system $O x_{1} y_{1} z_{1}$. The origin $O_{0}$ is on the horizontal plane, the axis $O_{0} X_{0}$ points to the initial navigation directions, the axis $o_{0} y_{0}$ points to top, the axis $O_{0} Z_{0}$ is determined by the right-hand-rule. The origin $O$ is on the centroid of the rigid body, the axis $O X$ points to head which coincides with the body symmetry axis, the axis oy is on the longitudinal plane which is vertical to the axis $O X$, the axis $O Z$ is determined by the right-hand-rule. The orgin $O_{1}$ coincides with the orgin $O$, the axis $O_{1} X_{1}$ coincides with the velocity vector of the orgin $o_{1}$, the axis $o_{1} y_{1}$ is on the longitudinal plane of the body and it is vertical to the axis $O_{1} X_{1}$, the axis $O_{1} z_{1}$ is determined by the right-hand-rule. $\theta, \psi, \varphi$ are defined to decribe the relationship between the inertial and the body coordinate system., $\alpha, \beta$ are defined to decribe the relationship between the speed and the body coordinate system. The meaning of the posture angles are same to the posture angles of torpedo and the underwater craft. 


\section{B. Dynamic equations}

In theory, when the towed system navigate under water, it will reach balance by the effect of hydrodynamic force, gravity, buoyancy and propeller thrust. According to the momentum theorem and the moment of momentum theorem, the sixdegrees-of-freedom equations of motion for the towed system can be expressed as:

$$
\left\{\begin{array}{c}
\frac{d \overrightarrow{Q_{t}}}{d t}+\vec{w} \times \overrightarrow{Q_{t}}=\vec{F} \\
\frac{d \overrightarrow{K_{t}}}{d t}+\vec{w} \times \overrightarrow{K_{t}}+\overrightarrow{v_{o}} \times \overrightarrow{Q_{t}}=\vec{M}
\end{array}\right.
$$

Where $\vec{F}$ denotes the external forces of the rigid, $\vec{M}$ denotes the net external torques of the rigid. The kinematics equations of the towed system can be expressed as:

$$
\begin{gathered}
\left(\begin{array}{c}
\dot{\theta} \\
\dot{\psi} \\
\dot{\varphi}
\end{array}\right)=\left(\begin{array}{c}
w_{y} \sin \varphi+w_{z} \cos \varphi \\
w_{y} \sec \theta \cos \varphi-w_{z} \sec \theta \sin \varphi \\
w_{x}-w_{y} \tan \theta \cos \varphi+w_{z} \tan \theta \sin \varphi
\end{array}\right) \\
\left\{\begin{array}{c}
v^{2}=v_{x}^{2}+v_{y}^{2}+v_{z}^{2} \\
\alpha=-\arctan \left(v_{y} / v_{x}\right) \\
\beta=\arctan \left(v_{z} / \sqrt{v_{x}^{2}+v_{y}^{2}}\right) \\
\left(\begin{array}{c}
v_{o x} \\
v_{o y} \\
v_{o z}
\end{array}\right)=C_{0}^{b}\left(\begin{array}{l}
v_{x} \\
v_{y} \\
v_{z}
\end{array}\right)
\end{array}\right.
\end{gathered}
$$

Where $v_{o x}, v_{o y}, v_{o z}$ are the velocity components of the rigid in the inertial coordinate system, $v_{x}, v_{y}, v_{z}, w_{x}, w_{y}, w_{z}$ are the velocity and the angular velocity components of the rigid in the body coordinate system, $C_{0}^{b}$ is the transformation matrix for the inertial and the body coordinate system. It has the same acceleration, velocity and displaceent at the connection point of rigids, while the forces have the same size and the opposite direction.

\section{SiMULATION EXAMPLE}

The towed system navigates straightly at the fixed depth 2.5 metres underwater, the velocity is $6 \mathrm{kn}$, and the length of the cable is 200 metres, the navigation body has control and power, the cable is recycled to the navigation body by $0.4 \mathrm{~m} / \mathrm{s}$ in relation to the navigation body. The coupling model above is used for numerical simulation and the results are shown below:

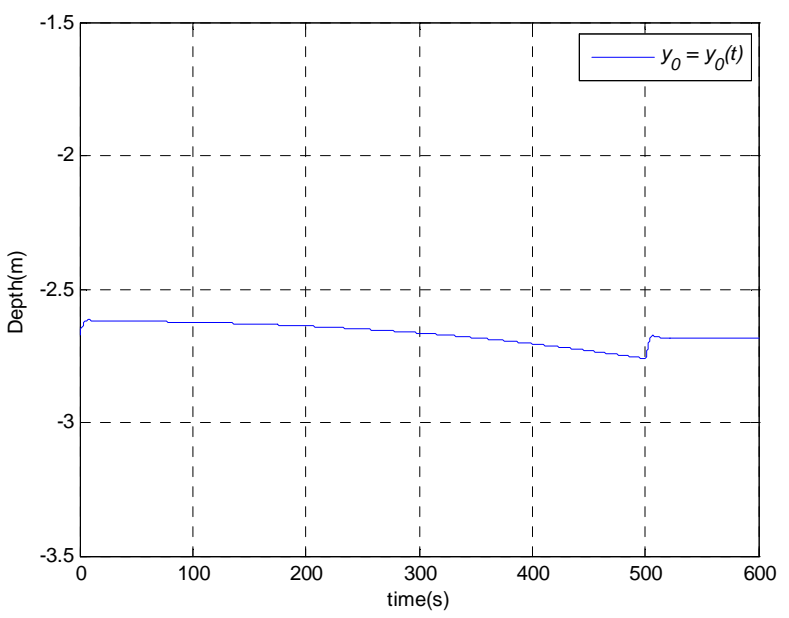

Figure 2. Time histories of the navigation body depth
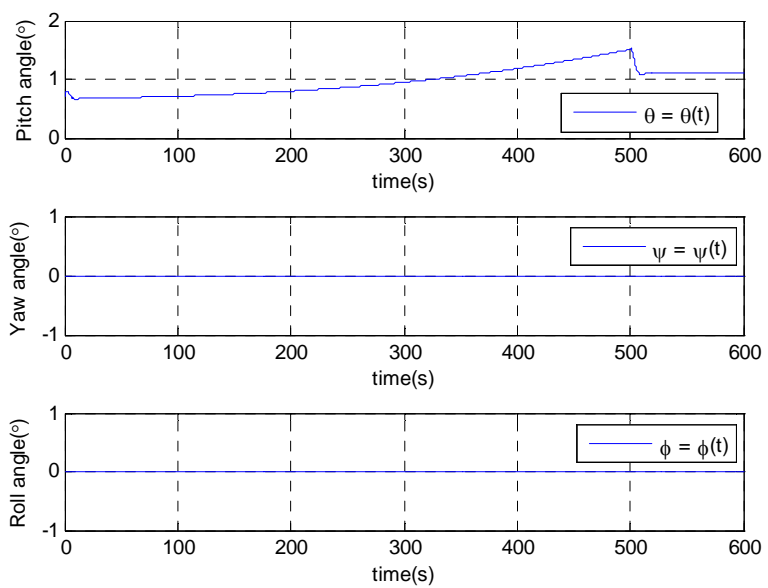

Figure 3. Time histories of the navigation body posture angle

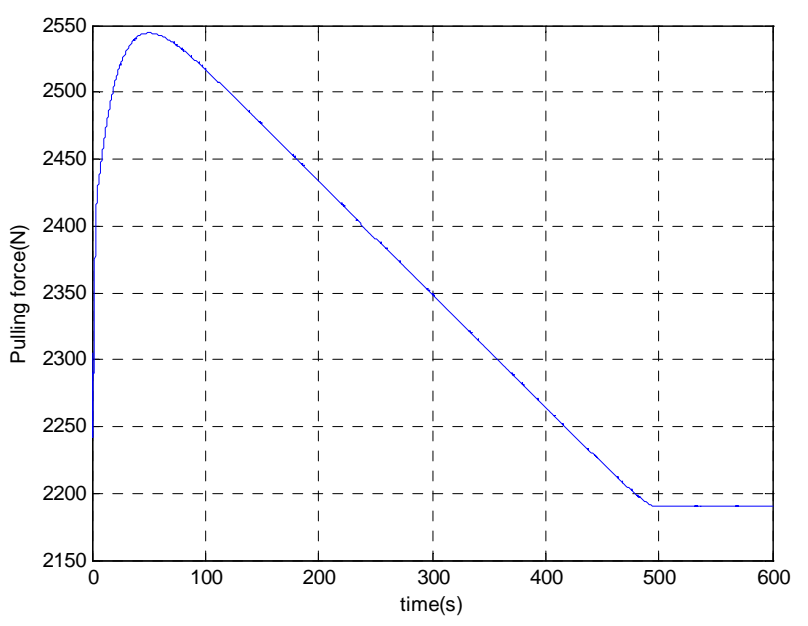

Figure 4. Time histories of the pulling force on the navigation body by the cable 


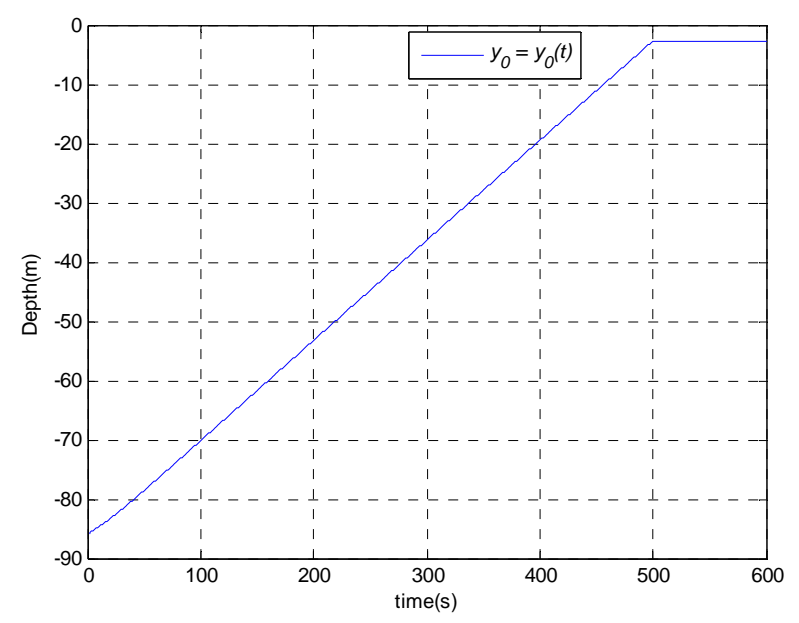

Figure 5. Time histories of the drag body depth
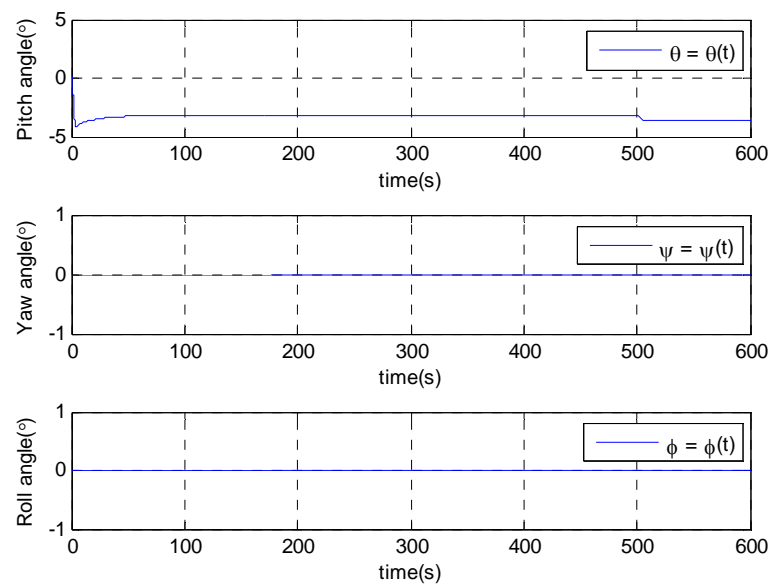

Figure 6. Time histories of the drag body posture angle

It can be seen that the motion of the navigation body,the cable and the drag body are coupling during the recycling; at the beginning of the recycling, due to the effect of the cable and the control the navigation body sinks slightly and the pitch angle of the navigation body increases until the cable is entirely recycled; the pulling force on the navigation body by the cable increases rapidly at the start and then decreases evenly in course of recycling; the drag body roses gradually and the pitch angle of the drag body keeps in a steady value.

\section{CONCLUSIONS}

A three-dimensions dynamic model of a typical underwater towed system is studied, and also the numerical simulation of the cable recycling is given which the towed system navigates at the fixed depth of 2.5 metres underwater by $6 \mathrm{kn}$ straightly. Via the figures shown above, it comes to the conclusions:

First, the motion of the navigation body , the cable and the drag body are coupling during the recycling;

Second, due to the pulling force by the cable, the navigation body sinks slightly, in order to navigate at the fixed depth, the control makes its' pitch angle increaseing, the system will get to steady until the cable is totally recycled;

Third, at the beginning of the recycling, the pulling force on the navigation body by the cable grows rapidly because of the start of recycling, after geting to the maximum value when the relative velocity get steady to $0.4 \mathrm{~m} / \mathrm{s}$, the force decreases gradually for the fluid resistance on the cable decreases greatly, the force will keep in a stable value when the cable is totally recycled;

At last, the depth of the drag body roses gradually and the pitch angle of the drag body keep in a stable value during the recycling, the depth and the pitch angle of the drag body will keep steady after the cable is completely recycled.

\section{REFERENCES}

[1] Ablow C M, Schechter S. Numerical simulation of under sea cable dynamics. Ocean Engineering, 1983, 10(6): 443-457.

[2] LIU Yu-qiang, SHENG Yan-feng, WANG Jun-qin,. Static Analysis and Attitude Calculation of Marine Submersible Buoy System and Its Application Development[J]. OCEAN TECHNOLOGY, 2010, 29 (3): 34-37..

[3] Cao Shengwen, Min Qiangli. Numerical Calculation Analysis of Ocean Submersible Buoy Mooring System[J]. MINE WARFARE \& SHIP SELF-DEFENCE, 2009, 17 (1): 27-30.

[4] Ranmuthugala, S.D., Gottschalk, S.A., 1993, Dynamic Simulation of a Two-Part Underwater Tow. Offshore Australia Confererce, 1-16. 